\title{
Diagnosis of Dental Cavities using Image Processing
}

\author{
Priyanca P. Gonsalves \\ Assistant Professor, \\ Department of Information Technology \\ St. Francis Institute of Technology, Mumbai
}

\begin{abstract}
Dental cavity is the disease inside the human mouth which is caused by different bacterial activities. Cavities make an everlasting damage in the tooth and it results in holes inside tooth. Dealing properly with dental cavities and taking an urgent treatment is always recommended to avoid more damage. Dentist recognizes the caries in patients' teeth by looking directly with eyes and sometimes with help of x-ray (radiograph) of teeth. The automated system would help the dentist to identify the caries in teeth by making use of x-ray. This paper proposes a model to detect the cavities using $\mathrm{x}$-ray images by making use of various image processing techniques, involving RGB to Gray conversion, generation of binary image, finding the region of interest, removing background, identifying regions and dividing image into multiple blocks and finally identifying the cavities present in $\mathrm{x}$-ray image.
\end{abstract}

\section{Keywords}

Dental caries, dental cavity, cavity detection, image processing, caries detection, $\mathrm{x}$-ray images, region detection.

\section{INTRODUCTION}

A human tooth is a structure made up of dentin, pulp and enamel. Mouth normally consists of various types of bacteria, they causes infection in human teeth. These infections generally termed as dental caries. Caries further damage the teeth permanently and results in tooth cavity. It is a very common disease found in world, about 60 to $90 \%$ of school children and nearly all adults have dental cavities [1]. Dental cavity affects the daily task of teeth by weakening the biting capacity, increased sensitivity, tooth ache, etc. Commonly when after meals, if the mouth is not washed properly, the food stays in corners of teeth, this deposited food generates acid. Such type of acid and sugar on teeth cover produces bacteria and it leads to breakdown of the tooth enamel (hard tissue of the teeth). Which causes caries in teeth, but if ignored at initial stage, caries can harm more neighboring teeth and go deeper inside the teeth till the pulp inside the teeth which can cause severe toothache.

Dentists normally treat their patients with caries by removing the radiographs (x-ray) of their tooth jaw and spot the cavities by observing the x-ray with naked eyes. This could lead to miss few cavities which are at early stage or which are not properly recognizable. Hence, there is a need of automation due to many reasons such as, lack of dental experts, different levels of expertise in each dentist and a second opinion is always helpful for confirm decision making.

This paper propose a novel technique to use combination of various image processing techniques, like RGB to Gray conversion, generation of binary image, finding the region of interest, removing background, identifying regions and dividing image into various blocks to extract the exact cavities from the $\mathrm{x}$-rays captured from patients tooth jaw and also speed up the operation. To diagnose the caries in teeth, best way of caries scanning is using an $\mathrm{x}$-ray; Dental x-ray clearly shows entire details of mouth which cannot be recognized while examining visually like hidden dental structures and bone loss [2]. The cavities in teeth can be identified uniquely from x-ray images because; discontinuity in the brightness of an x-ray corresponds to discontinuity in depth, surface orientation, material property and variation in scene illumination. Cavity properties are different than properties of a healthy tooth [3]. The $\mathrm{x}$-rays need to undergo required image processing techniques. The captured $\mathrm{x}$-ray is in jpeg format thus, it is in RGB format of color code. The image is then converted to gray color code where the pixel values of image are not changed. After converting to gray color, feature enhancement is applied to highlight the affected part (cavity in teeth) followed by background removal and then region and block analysis and finally caries detection.

The entire paper is organized in stepwise format first part is introduction about the cavities and how to overcome it using Image Processing. Section 2 covers, the analysis performed on cavities handling. Sections 3 elaborate more on the working of the proposed work. Section 4 concludes the proposed concept and suggests the future work possible.

\section{ANALYSIS}

Dental caries is a disease for which the dentist needs a final confirmation on existence of cavity in his patients' teeth. Scanning of x-ray is the important part on which the final decision is executed. X-ray scanning needs to use various image processing techniques. Image processing consists of a collection of huge methods and algorithms.

One of the treatments used for this cause is proposed by, Villette, A., et al. In their study, they describe a new device for the rapid and painless injection of a calcium hydroxide paste under controlled pressure in the tooth pulp cavity with a controlled volume of the paste. They observed that the reduction of the volume of the pulp cavity and that the extent of the tooth devitalization depend on the amount of the paste injected [4].

There is also a technique that is making use of ultrasound concept. The researchers have done experimental evaluation of human teeth using noninvasive ultrasound. It is verified that these experimental measurements confirm predictions reported in earlier finite element and transmission line studies and suggest that ultrasound is a tool is helpful and can result in better improvement in current dental imaging systems [5].

Other than cavity detection, leaf disease detection also depends on captured leaf images. Thus, it makes use of image processing. Francis, Jobin, and B. K. Anoop have proposed an algorithm for detection and identification of leaf disease using image processing. The pepper plant leaves are used here, these leaves undergo image segmentation process in two phases, masking process and threshold based segmentation. 
This algorithm produces better results in terms of differentiating healthy and unhealthy leaves [6].

Dandawate Yogesh and Radha Kokare have Used support vector machine to classify soybean leaves as healthy or diseased [7].

Thus, image processing plays an important role in performing cavity detection along with the supporting image processing techniques like image conversion, preprocessing,

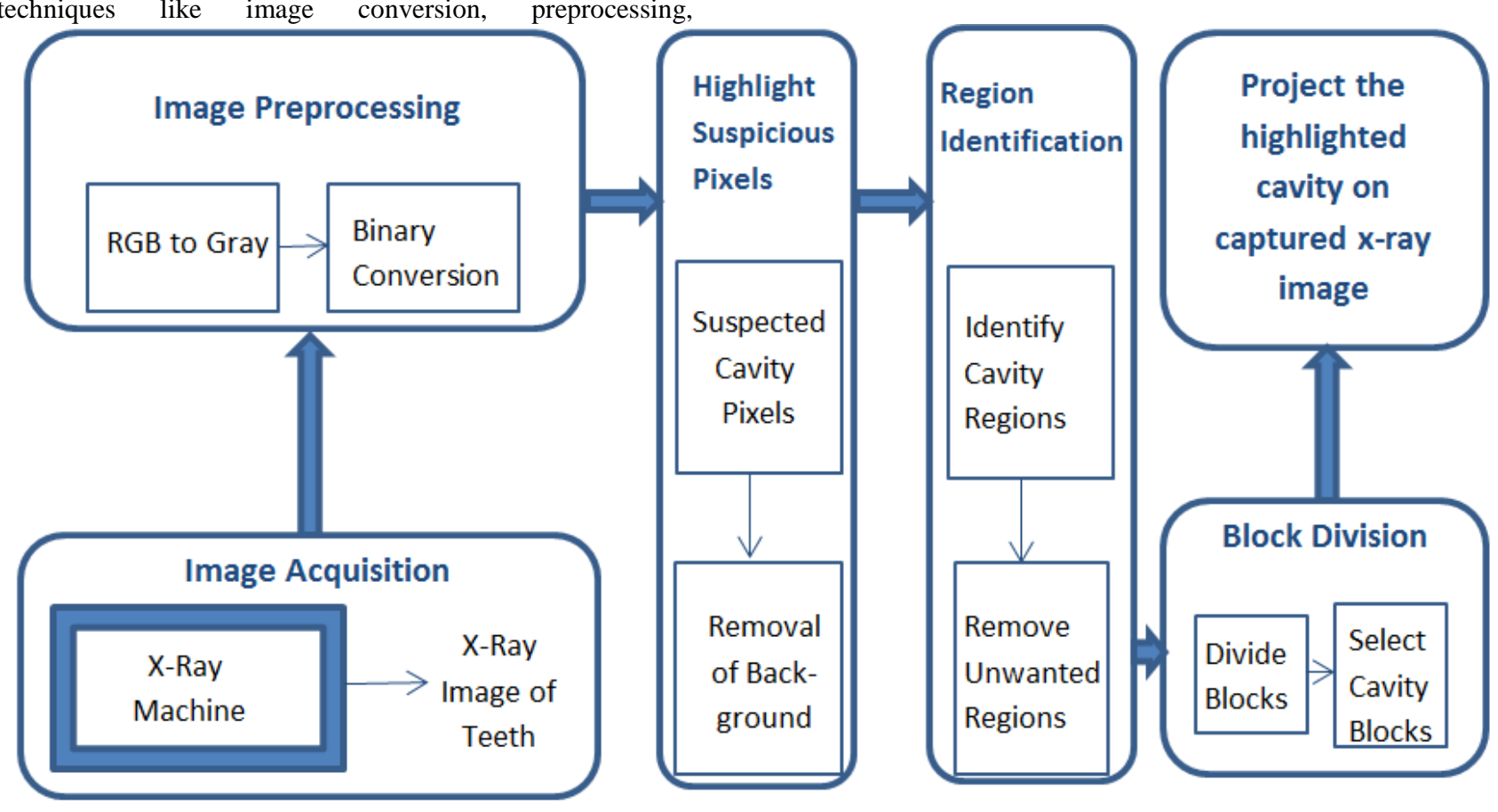

Figure 1: Block diagram of proposed work

\subsection{Image Acquisition}

The dentists normally don't use any tools for finding the cavities in teeth. Caries are not seen easily with eyes; It can be recognized effectively only with the use of $x$-ray images of teeth.

In x-ray, a healthy tooth is normally seen in white color and other area such as soft tissue, jaw and mouth are seen in dark shades. The cavities and caries are also seen with specific shades and structure. X-ray images when captured, they might have lots of different shades from bright to dark formed due to light at x-ray center or the position of teeth from x-ray plate. Digital images can be affected by various types of noise [8]. Various types of noise found are Gaussian noise, salt and pepper noise, white noise, etc. [9]. For better quality of images that is avoiding noise; $x$-ray should be captured with proper care and attention. Below Fig. 2 is the original $\mathrm{x}$-ray image used as an input for the model. segmentation, region identification, block division.

\section{WORKING}

The proposed model for cavity detection using Image Processing is explained below with the following block diagram. Fig. 1 illustrates the working of the proposed model. Each block from this diagram is elaborated further with proper explanation.

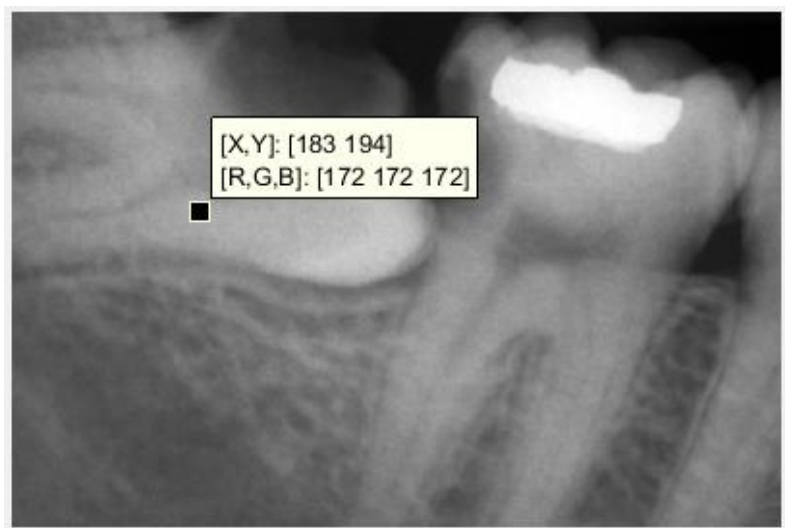

Figure 2: Original $x$-ray image of human teeth in RGB format

\subsection{Image Preprocessing}

Image Preprocessing is a collection of operations performed for enhancing the image data and removing unwanted noise and background data. Following subsections explain image pre-processing steps in detail.

\subsubsection{RGB to Gray Conversion}

The captured x-ray images are saved in jpeg format. These images are very similar to gray images; that is, almost all pixels in entire image have all three colors Red, Green and Blue hold same intensity value. The x-ray images are thus converted to gray images for saving storage space and 
processing speed.

Above figure 2 after converting from RGB to Gray is shown as below in Fig. 3 .

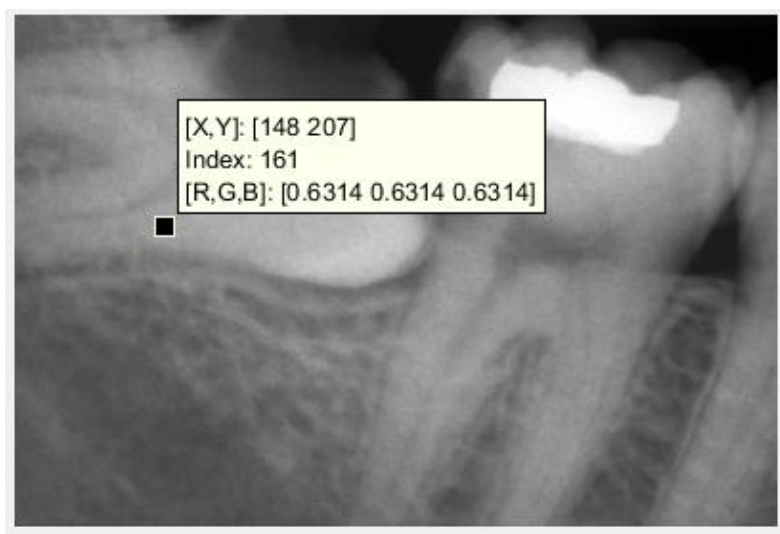

Figure 3: Gray scale image of teeth

\subsubsection{Binary Conversion}

Gray color x-ray images are converted to binary images. A binary image has basically two colors only, black and white. This binary image is then compared with gray image of same sample and the black portion of image is trimmed down from original gray color x-ray image. Following Fig. 4 shows the binary image of above image shown in Fig. 3.

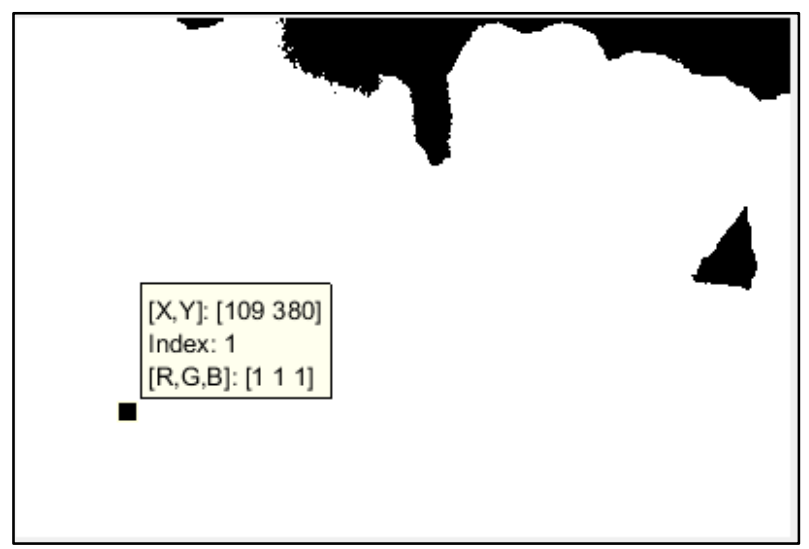

Figure 4: Binary image of teeth

\subsection{Highlight Suspicious Cavity Pixels}

Image preprocessing step is followed by the classification step; in this step, the pixel values of cavities are accepted from dataset of dental x-ray images.

\subsubsection{Suspected Cavity Pixels}

The pixel's values are classified in two classes one is named 'class 1' (indicates class of cavity pixels) and remaining pixels are classified under 'class 2' (indicates class of noncavity pixels). The image after highlighting the suspected cavities is given as follows in Fig.5.

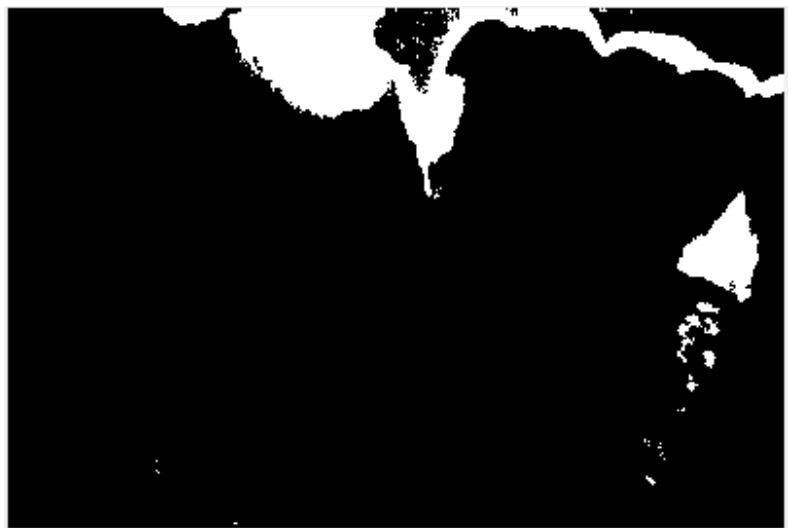

Figure 5: Suspected Cavities identified in range

\subsubsection{Removal of Background}

The cavity pixel color and background colors falls in nearly same range. Removal of background part from image, indirectly ease the process of identifying the cavities present in $\mathrm{x}$-ray. The human teeth are selected as region of interest (ROI) very first. The process of extracting ROI from given $\mathrm{x}$ ray is followed by generation of binary image. The above image in figure- is displaying cavities, but here some part of the background is wrongly shown as cavity. The background from the above image in Fig. 5 is removed with the use of binary image shown in Fig. 4; the resultant image is displayed below in Fig. 6.

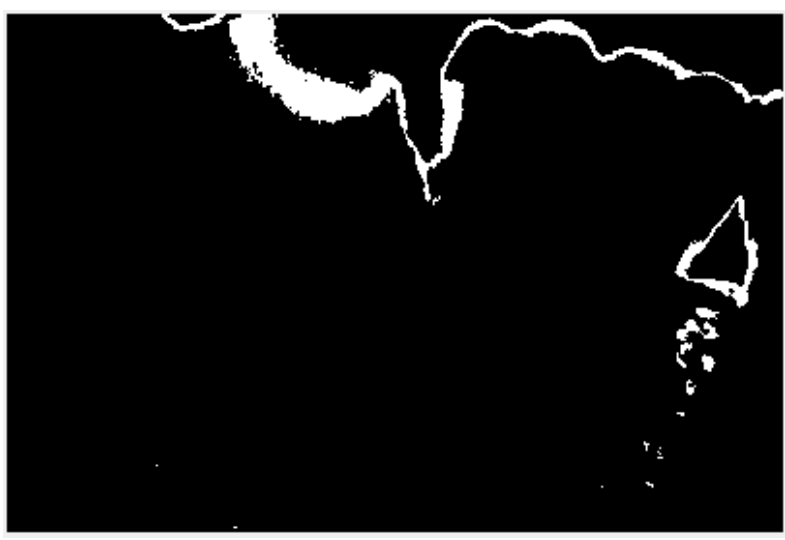

Figure 6: Suspected Cavities after removing background

\subsection{Region Identification}

\subsubsection{Identify Cavity Regions}

The cavity pixels highlighted in the image shown in Fig. 7 are represented in regions with proper outline. These regions are further examined and the regions centroid and circular area is observed. 


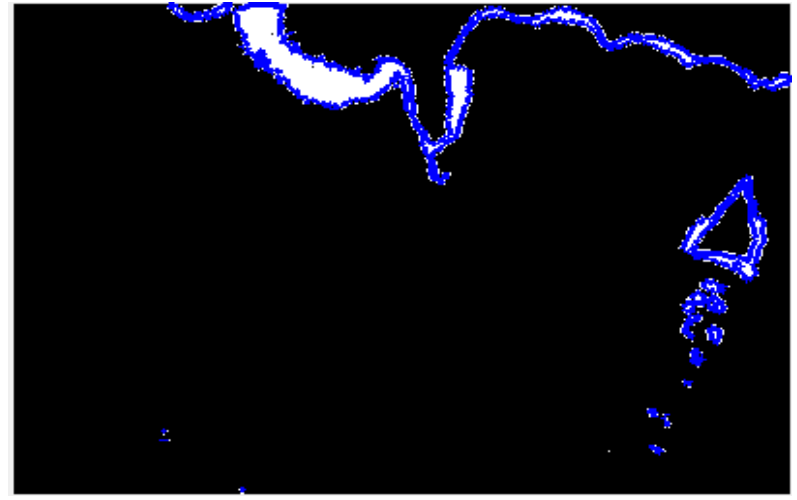

Figure 7: Cavities represented with regions

\subsubsection{Remove Unwanted Regions}

All the identified regions are further examined and then by making use of Major Axis Length and Minor Axis Length, some of the regions are removed which doesn't fall in category of true cavity regions. Following Fig. 8 displays the selected regions with their centroids.

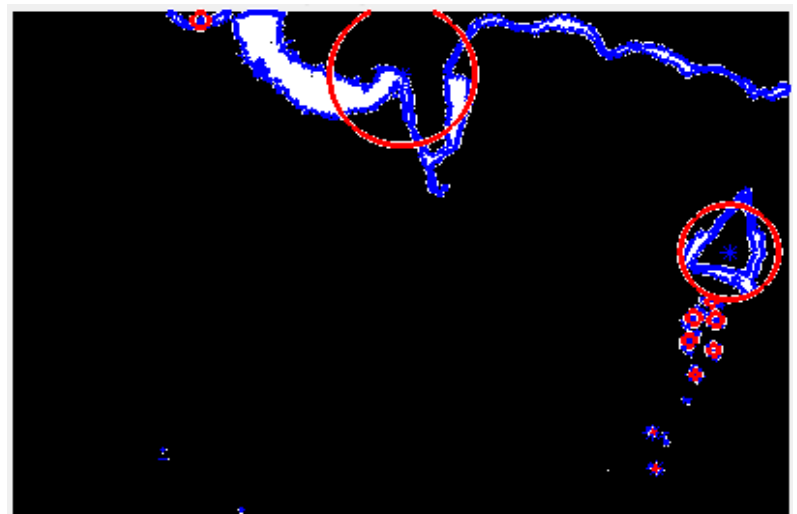

Figure 8: Centroids of cavity regions after removing unwanted regions

\subsection{Block Division}

\subsubsection{Divide image into Blocks}

The cavity regions identified till now in Fig. 8 are long enough, which are not so easy to evaluate to identify the true cavity. The image is further divided into blocks of similar sizes (50 X 50 pixels).

\subsubsection{Select Cavity Blocks}

The blocks are now cross checked to find the number of cavity pixels in each of them. Here the blocks which are showing false cavity that is edge of teeth; are eliminated and only true cavity in which number of cavity pixels are large in numbers are selected.
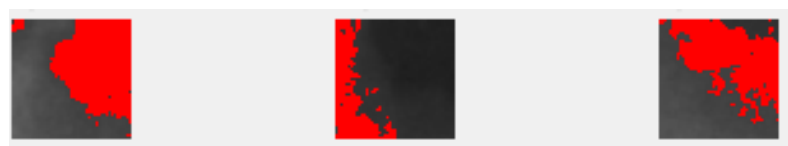

Figure 9: Selected blocks after removing unwanted blocks

Above Fig. 9 displays the blocks that are selected as the block with true cavity region. In these blocks, the red region is representing cavity area and other portion is representing healthy area.

\subsection{Output}

The selected blocks with true cavity are now used to generate final output image, in which the cavity pixels are shown with highlighted color. The output image generated by this model is very useful for dentists to easily identify the cavity existing in the x-ray of teeth.

Below image in Fig. 10 is the original $\mathrm{x}$-ray image which is used as an input to the model.

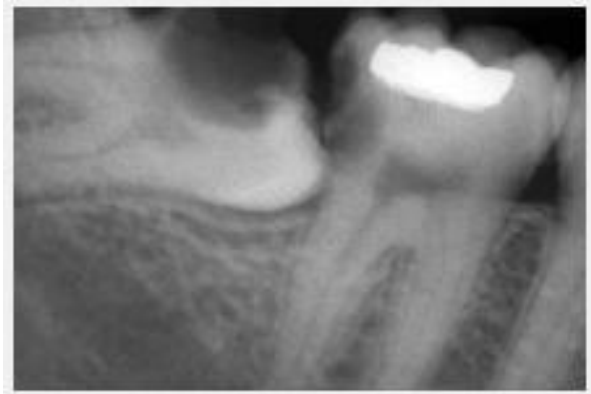

Figure 10: Original X-ray image

Following image in Fig. 11 is the final resultant image which shows cavity portion in highlighted area that is helpful for dentists to easily identify the cavity from an x-ray image.

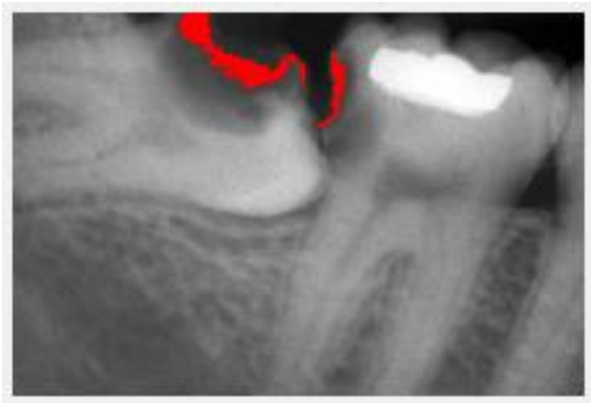

Figure 11: Final result showing cavities on original X-ray image

\section{CONCLUSION}

Dental cavity is a widespread disease that affects many people worldwide. Due to human dependency the caries can sometimes be ignored. This paper proposes a model to easily identify the cavities in teeth by availing the potential of Image Processing.

Many of the Image Processing functions used are RGB to Gray conversion, generation of binary image, finding the region of interest, removing background, identifying regions and dividing image into same sized blocks and finally coming up with identifying the cavities present in $\mathrm{x}$-ray image with proper position.

In future, cavity detection work can be further improvised by using the final identified blocks as input to ANN model with backpropagation algorithm. Supervised learning of ANN helps to generate patterns by itself from training dataset. The trained ANN can be later tested and validated. Finally the model can be used for new unseen samples of dental x-ray to detect the cavities.

\section{ACKNOWLEDGMENTS}

Special thanks to, Abdolvahab Ehsani Rad for sharing a dataset of 120 digital periapical X-ray radiograph images that helped in implementation of the above mentioned research [10-13]

\section{REFERENCES}

[1] http://www.who.int/mediacentre/factsheets/fs 318/en/ World Health Organization 
[2] A. K. Jain and H. Chen, "Matching of dental x-ray images for human identification", Pattern Recognition, 37:1519-1532, 2004.

[3] Wang, X.Q. Ye, W.K. Gu, "Training a Neural Network for Moment Based Image Edge Detection" Journal of Zhejiang University SCIENCE(ISSN 1009-3095, Monthly), Vol.1, No.4, pp. 398-401 CHINA, 2000.

[4] Villette, A., et al. "Pulp tissue response to partial flling of the pulp cavity, under compression, by calcium hydroxide, using a new device." Engineering in Medicine and Biology Society, 1992 14th Annual International Conference of the IEEE. Vol. 3. IEEE, 1992.

[5] Ghorayeb, Sleiman R., and Teresa Valle. "Experimental evaluation of human teeth using noninvasive ultrasound: echodentography." ieee transactions on ultrasonics, ferroelectrics, and frequency control 49.10 (2002): 14371443.

[6] Francis, Jobin, and B. K. Anoop. "Identification of leaf diseases in pepper plants using soft computing techniques." Emerging Devices and Smart Systems (ICEDSS), Conference on. IEEE, 2016.

[7] Dandawate, Yogesh, and Radha Kokare. "An automated approach for classification of plant diseases towards development of futuristic Decision Support System in Indian perspective." Advances in Computing,
Communications and Informatics (ICACCI), 2015 International Conference on. IEEE, 2015.

[8] K. M. Rao, Deputy Director, NRSA Hyderabad, "Overview of image processing", Readings in Image processing.

[9] Gonzalviz R. C., Woods R. E.,"Digital Image Processing", Pearson Publications, 2009.

[10] Abdolvahab Ehsani Rad, Mohd Shafry Mohd Rahim, Amjad Rehman, and Tanzila Saba. Digital dental x-ray database for caries screening. 3D Research, 7(2):1-5, 2016.

[11] Rad, A. E., Mohd Rahim, M. S., Rehman, A., Altameem, A., \& Saba, T. (2013). Evaluation of current dental radiographs segmentation approaches in computer-aided applications. IETE Technical Review, 30(3), 210-222.

[12] Abdolvahab Ehsani Rad, Mohd Shafry Mohd Rahim, Hoshang Kolivand, and Ismail Bin Mat Amin. Morphological region-based initial contour algorithm for level set methods in image segmentation. Multimedia Tools and Applications, pages 1-17, 2016.

[13] Rad, A. E., Amin, I. B. M., Rahim, M. S. M., \& Kolivand, H. (2015). Computer-Aided Dental Caries Detection System from X-Ray Images. In Computational Intelligence in Information Systems (pp. 233-243). Springer International Publishing. 